\title{
Development and validation of a novel MR imaging predictor of response to induction chemotherapy in locoregionally advanced nasopharyngeal cancer: a randomized controlled trial substudy (NCT01245959)
}

Di Dong ${ }^{1,2,3+}$, Fan Zhang ${ }^{2,4+}$, Lian-Zhen Zhong ${ }^{1,3+}{ }^{\text {, Meng-Jie Fang }}{ }^{1,3}$, Cheng-Long Huang ${ }^{2}$, Ji-Jin Yao ${ }^{4}$, Ying Sun ${ }^{2}$, Jie Tian ${ }^{1,5^{*}}$, Jun $\mathrm{Ma}^{2^{*}}$ and Ling-Long Tang ${ }^{2^{*}}$

\begin{abstract}
Background: In locoregionally advanced nasopharyngeal carcinoma (LANPC) patients, variance of tumor response to induction chemotherapy (ICT) was observed. We developed and validated a novel imaging biomarker to predict which patients will benefit most from additional ICT compared with chemoradiotherapy (CCRT) alone.

Methods: All patients, including retrospective training $(n=254)$ and prospective randomized controlled validation cohorts (a substudy of NCT01245959, $n=248$ ), received ICT+CCRT or CCRT alone. Primary endpoint was failure-free survival (FFS). From the multi-parameter magnetic resonance images of the primary tumor at baseline, 819 quantitative 2D imaging features were extracted. Selected key features (according to their interaction effect between the two treatments) were combined into an Induction Chemotherapy Outcome Score (ICTOS) with a multivariable Cox proportional hazards model using modified covariate method. Kaplan-Meier curves and significance test for treatment interaction were used to evaluate ICTOS, in both cohorts.

(Continued on next page)
\end{abstract}

\footnotetext{
* Correspondence: tangll@sysucc.org.cn; tangll@mail.sysu.edu.cn; jie.tian@ia.ac.cn; majun2@mail.sysu.edu.cn

${ }^{\dagger}$ Di Dong, Fan Zhang and Lian-Zhen Zhong contributed equally to this work. 'CAS Key Laboratory of Molecular Imaging, Institute of Automation, Chinese Academy of Sciences, No. 95 Zhongguancun East Road, Hai Dian District, Beijing 100190, People's Republic of China

${ }^{2}$ Department of Radiation oncology, State Key Laboratory of Oncology in South China; Collaborative Innovation Center for Cancer Medicine, Sun Yat-sen University Cancer Center, 651 Dongfeng Road East, Guangzhou 510060, People's Republic of China

Full list of author information is available at the end of the article
}

(c) The Author(s). 2019 Open Access This article is distributed under the terms of the Creative Commons Attribution 4.0 International License (http://creativecommons.org/licenses/by/4.0/), which permits unrestricted use, distribution, and reproduction in any medium, provided you give appropriate credit to the original author(s) and the source, provide a link to the Creative Commons license, and indicate if changes were made. The Creative Commons Public Domain Dedication waiver (http://creativecommons.org/publicdomain/zero/1.0/) applies to the data made available in this article, unless otherwise stated. 
(Continued from previous page)

Results: Three imaging features were selected and combined into ICTOS to predict treatment outcome for additional ICT. In the matched training cohort, patients with a high ICTOS had higher 3-year and 5-year FFS in ICT+CCRT than CCRT subgroup (69.3\% vs. $45.6 \%$ for 3 -year FFS, and $64.0 \%$ vs. $36.5 \%$ for 5 -year FFS; $\mathrm{HR}=0.43,95 \% \mathrm{Cl}=0.25-0.74, p=0.002$ ), whereas patients with a low ICTOS had no significant difference in FFS between the subgroups $(p=0.063)$, with a significant treatment interaction ( $\left.p_{\text {interaction }}<0.001\right)$. This trend was also found in the validation cohort with high $(n=$ 73, ICT+CCRT $89.7 \%$ and $89.7 \%$ vs. CCRT $61.8 \%$ and $52.8 \%$ at 3-year and 5 -year; $\mathrm{HR}=0.17,95 \% \mathrm{Cl}=0.06-0.51, p<0.001$ ) and low ICTOS $(n=175, p=0.31)$, with a significant treatment interaction ( $\left.p_{\text {interaction }}=0.019\right)$. Compared with $12.5 \%$ and 16.6\% absolute benefit in the validation cohort (3-year FFS from 69.9 to $82.4 \%$ and 5-year FFS from 63.4 to $80.0 \%$ from additional ICT), high ICTOS group in this cohort had 27.9\% and 36.9\% absolute benefit. Furthermore, no significant survival improvement was found from additional ICT in both groups after stratifying low ICTOS patients into low-risk and high-risks groups, by clinical risk factors.

Conclusion: An imaging biomarker, ICTOS, as proposed, identified patients who were more likely to gain additional survival benefit from ICT+CCRT (high ICTOS), which could influence clinical decisions, such as the indication for ICT treatment.

Trial registration: ClinicalTrials.gov, NCT01245959. Registered 23 November 2010.

Keywords: Individualized imaging biomarker, Induction chemotherapy, Survival benefit, Treatment decision, Locoregionally advanced nasopharyngeal cancer

\section{Introduction}

Nasopharyngeal carcinoma (NPC) has a unique geographical distribution; 86,700 new cases of nasopharyngeal carcinoma were reported worldwide in 2012 with the highest incidence reported in Southeast Asia [1]. Nearly 70\% of newly diagnosed cases of NPC are classified as locoregionally advanced disease [2]. Induction chemotherapy (ICT) followed by concurrent chemoradiotherapy (CCRT) or CCRT alone are both now recommended for locoregionally advanced NPC (LANPC) in the National Comprehensive Cancer Network (NCCN) guideline [3]. Our previous prospective multi-center randomized controlled trial showed that the addition of ICT to CCRT yielded 8-9\% improvement in failure-free survival (FFS) in LANPC [46]. However, compared to CCRT, extra grade 3 or 4 adverse events, such as neutropenia and leucopenia, were $>40 \%$ during ICT [5]. Further analysis of this trial showed that variance of tumor response to ICT was observed in different patients; while $9.1 \%$ of patients presented with poor tumor response (stable disease, SD), 11.3\% showed complete response (CR) [7]. Limited benefit and obvious toxicity from ICT and differences in response to ICT indicated the need for an individualized biomarker to predict which patients will benefit most from additional ICT.

Tumors are recognized as heterogeneous entities, and the realization that distinct molecular subsets exist requires a shift in cancer treatment development, from a "one-size fits all" to a more personalized and group-based treatment design [8]. Great efforts have been made to search for molecular biomarkers for LANPC, such as plasma EpsteinBarr virus (pEBV)-DNA [9] and miRNAs [10]. However, few biomarkers are widely used as a predictive tool for personalized therapy in clinical practice, and there is therefore an urgent need to identify new biomarkers. As a promising research method for tumor heterogeneity, the role of medical imaging is swiftly evolving from being primarily a diagnostic tool to also include a central role in assisting with individual treatment decision [11].

Radiomics is an emerging technique that can deeply analyze tumor phenotype by converting medical images into minable data and extracting thousands of quantitative features as imaging biomarkers [11]. Noninvasive radiomic features could reflect the pathologic, genetic, and prognostic information of the entire tumor and thus assist with diagnosis and prognosis [12, 13]. Evidence from previous studies showed that radiomic features could predict treatment outcome and screen out patients for individual treatment [14]. For this reason, radiomics provides a possible way to develop clinical decision support systems (CDSSs), individual information-based systems designed to generate patient-specific recommendations, for example, whether a patient is suitable for ICT+CCRT or CCRT alone.

In this study, we aimed to use pretreatment magnetic resonance (MR) images and clinical characteristics of NPC from a retrospective cohort to develop an individualized radiomic biomarker, to predict which patients will benefit most from additional ICT. Then, we used a subset from a randomized controlled trial to validate the performance of the biomarker and to recommend a CDSS the best choice between ICT+CCRT and CCRT alone in LANPC.

\section{Methods}

\section{Study design and participants}

In this study, we included a retrospective cohort for training, and a prospective randomized controlled cohort for validation, all from Sun Yat-sen University Cancer Center (Guangzhou, China). The validation cohort was a subset of our previous multi-center, open-label, stratified 
randomized phase 3 controlled trial (ClinicalTrials.gov identifier NCT01245959) comparing ICT+CCRT and CCRT alone in patients between March 2011 and August 2013 [5], which was an intention-to-treat population. For the training cohort, we retrospectively retrieved medical records of LANPC patients who received radical ICT+CCRT or CCRT between 2008 and 2016. Patients who met the inclusion and exclusion criteria and treatment, in accordance with our previous open-label, phase 3 randomized controlled trial, were recruited in the training cohort (detailed in Additional file 1). The authenticity of this study has been validated by uploading the key raw data onto the Research Data Deposit public platform (www.researchdata.org.cn), with the approval RDD number as RDDA2019001070. Finally, 254 and 248 patients were included in the training and validation cohorts, respectively.

The ethical review board of our institution approved this retrospective analysis of anonymous data, and the requirement for informed consent was waived. All patients underwent pretreatment multi-parametric magnetic resonance imaging (MRI) within 2 weeks before any anti-cancer treatment. Non-contrast enhanced MRI (T1-weighted images in axial, coronal, and sagittal planes, as well as axial T2-weighted images) and contrast-enhanced sequence (axial and sagittal T1weighted images, as well as coronal T1-weighted images) were performed sequentially for each patient. Detailed MR protocols (version of MR scanner, magnet strengths, contrast agents, image thickness, etc.) are shown in Additional file 2, Additional file 10: Table S1, and Additional file 11: Table S2.

The radiotherapy and chemotherapy treatment details have been reported previously [5]. After treatment, patients were assessed every 3 months during the first 3 years, and every 6 months thereafter. The primary endpoint was FFS (time to locoregional failure, distant failure, or death from any cause, whichever occurred first). The secondary endpoints included overall survival ([OS], time to death from any cause), distant FFS ([D-FFS], time to distant failure), and locoregional FFS ([LR-FFS], time to local or regional failure or both).

Before the model construction, there should be no subjective judgment by doctors, and patients should have equal chance to choose ICT+CCRT or CCRT alone, when using observational data to evaluate the treatment effects. Therefore, we used Inverse probability of treatment weighting (IPTW) to balance the baseline differences in the training cohort [15]. IPTW was performed on the basis of age, sex, pEBV DNA, cervical nodal necrosis, primary tumor volume, $\mathrm{N}$ stage, and $\mathrm{T}$ stage (detailed description is shown in Additional file 3). Matched patients were included in the analysis, as the matched training cohort.

\section{Procedures}

The radiomics workflow is shown in Fig. 1. All MR images were retrieved from the picture archiving and communication system (PACS) and exported to the ITKSNAP software (version 3.4.0; www.itksnap.org) for manual segmentation. The region of interest (ROI) was delineated manually in the primary tumor on each slice of the axial T1-weighted, T2-weighted, and contrast-enhanced T1-weighted images. Coronal and sagittal images were only used to guide the cross-sectional segmentation of the ROIs. Therefore, there were three different ROIs segmented for each patient in this study. A radiologist with 10 years of experience with MR (L.L.T.) performed all image segmentations. To evaluate the reproducibility of feature among segmentations by different radiologists (inter-observer) or one radiologist at different times (intra-observer), 30 patients were randomly selected 30 days after the initial segmentation and their images were segmented again by L.L.T. and another radiologist (F.Z.) in the same way. All the radiologists were blinded to treatment group and patient outcome.

After segmentation, interpolation and normalization of MRI images were performed to control inter-scanner and inter-vendor variability of features (detailed in Additional file 3). Then, 273 2D radiomic features were extracted from each ROI, and a total of 819 2D features were achieved per patient. All the features have been referred to in the image biomarker standardization initiative (IBSI) formula [16] and in the study by Aerts et al. [17]. These features were derived from the tumor image intensity, shape, gray-level co-occurrence matrix (GLCM), and graylevel run-length matrix (GLRLM) (Additional file 3) [16, 17]. The process of feature extraction was conducted using Matlab 2017b (Mathworks, Natick, MA, USA). Radiomic features of all patients were standardized by the $z$-score method, based on parameters calculated from the training cohort. Then, the intra-observer/inter-observer agreements of radiomic features were assessed using the inter-class correlation coefficient (ICC) on the 30 patients' multiple segmentation data [18]. For the intra-observer agreement, the ICC of each radiomic feature was calculated between the twice segmentations by L.L.T. For the inter-observer agreement, the ICC of each radiomic feature was calculated between segmentations by L.L.T. and F.Z. The features with ICCs $>0.8$ in both intra-observer and inter-observer tests were reserved.

To construct the predictive model, we first identified which factors could provide different prognostic information in the ICT+CCRT and CCRT alone groups. Using the matched training cohort, the interaction term of treatment and each radiomic feature was ranked by its univariate $p_{\text {interaction }}$ value in a Cox proportional hazard model. Radiomic features were excluded when their correlation coefficient with the former ranked features 

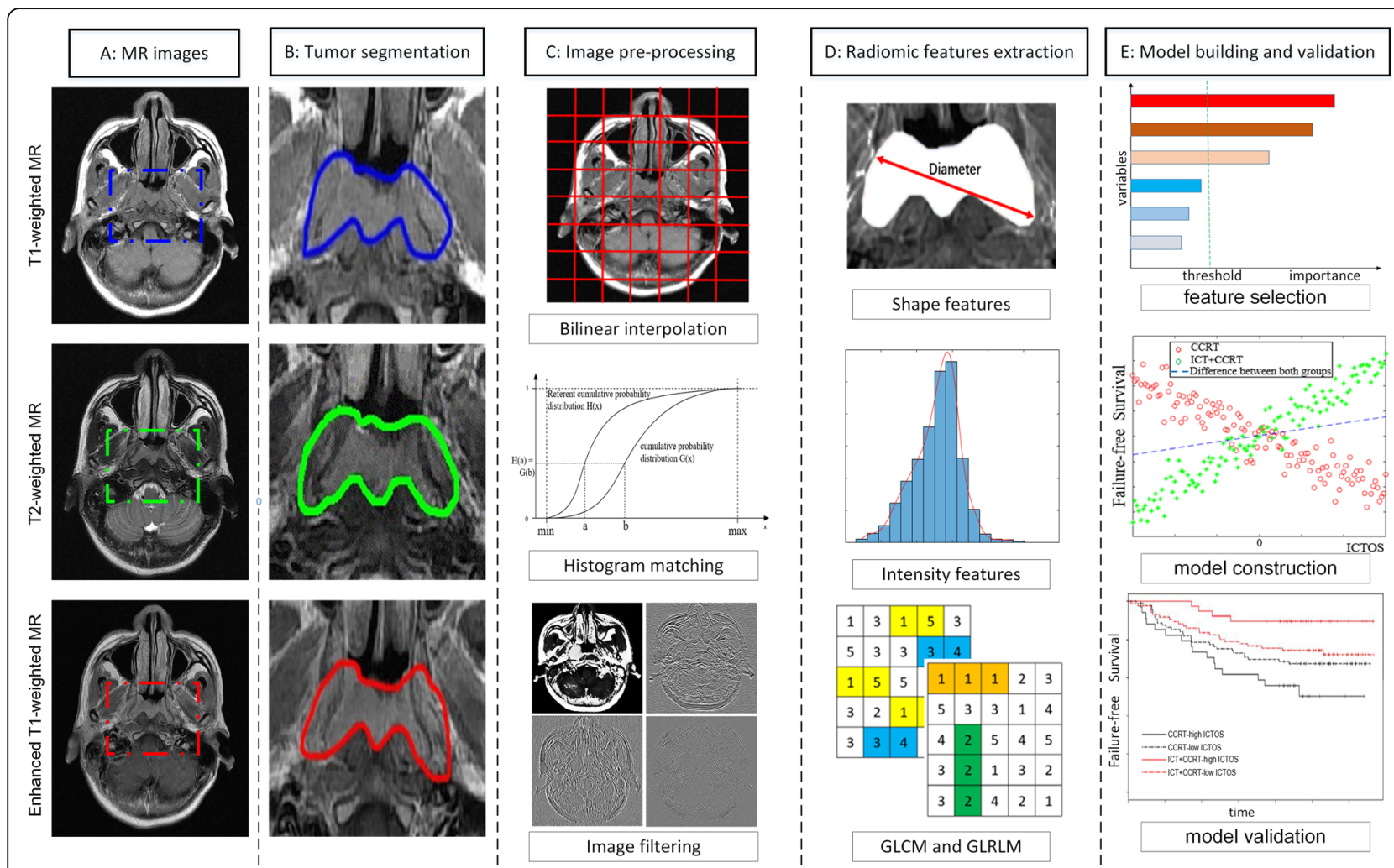

Fig. 1 Radiomics workflow in this study. a Collection of multi-sequence MR images. b Tumor segmentation by radiologists. $\mathbf{c}$ Preprocessing of the MR images. $\mathbf{d}$ Feature extraction from tumor region. e Model construction and validation

was $>0.8$, to reduce redundancy between radiomic features. The remaining radiomic features were used to train a multivariable Cox proportional hazard model, with FFS as the endpoint, using the modified covariate method [19]. Backward step-wise selection was used to select variables whose coefficients reached statistical significance. The outcome score of the model, termed Induction Chemotherapy Outcome Score (ICTOS), was calculated using the difference in treatment outcome between ICT+CCRT and CCRT alone. Patients with scores $>0$ (high ICTOS) benefit from additional ICT, while patients with scores $\leq 0$ (low ICTOS) do not benefit from additional ICT. The "0" cutoff point represents the no difference point in predicted outcomes for additional ICT. Stratified analyses for low ICTOS patients were performed to test the ICTOS's robustness in various subgroups of the validation dataset and find those patients who need further treatment.

\section{Statistics}

We aimed to identify patients who will benefit the most, of the entire cohort, defined as the population who would have a 5-year FFS rate increase from 65\% (with CCRT alone) to $90 \%$ (with ICT+CCRT) (target HR < 0.25 ) [4]. The statistical methodology is as reported by Zhao et al. [20]. With 110 patients and 29 events, 1:1 assigned to each arm and 5\% two-sided statistical significance, the power for the superior test was 0.96 . Assuming this population accounts for $47 \%$ of LANPC [21], 234 patients were required for the training cohort.

Kaplan-Meier curves were generated for FFS (the primary endpoint). All hazard ratios (HRs), 3-year FFS, and 5-year FFS were reported with $95 \%$ confidence intervals (CIs). We used the Greenwood's formula to obtain the $95 \%$ CI for 3year and 5-year FFS. Wald test was used to assess the significance of the interaction term while log-rank test was used to compare the different survival curves.

Continuous variables were expressed as median (range), and group comparison was performed by either the $t$ test or the Wilcoxon rank-sum test. Categorical variables were expressed as percentages, and group comparison was performed by either the Pearson $\chi^{2}$ test or the Fisher exact test. Statistical analysis was conducted with $\mathrm{R}$ software (version 3.4.3; http://www.Rproject.org). Detailed description of the modified covariate method is shown in Additional file 3. A two-sided $p$ value $<0.05$ was used to indicate statistical significance. Note that only absolute benefits in this manuscript were expressed as percentage points, and all others were expressed as percentages.

\section{Results}

Finally, 254 and 248 patients (from the initial 263 and 257 eligible patients) were included in the training and 
validation cohorts, respectively (Fig. 2). After IPTW, the ICT+CCRT and CCRT alone groups achieved good balance in the matched training cohort (matched sample size $n=247.8$ ). The characteristics of the matched training cohort are shown in Additional file 12: Table S3 and Additional file 5: Figure S1. In the matched training and validation cohorts, respectively, $39.4 \%$ and $28.6 \%$ patients had treatment failure or died.

After the intra-observer/inter-observer agreement assessment, 689 radiomic features with ICC $\geq 0.8$ were reserved for subsequent analysis (Additional file 6: Figure S2). In the matched training cohort, three radiomic features including intensity (skewness) and two texture (variance from Gray-Level Co-occurrence Matrix [GLCM_variance] and Long Run High Gray Level Emphasis from Gray-Level Run-Length matrix [GLRLM LRHGLE]) features were selected and incorporated into the ICTOS model: $-0.668 *$ skewness - $0.442 *$ GLCM_variance + 0.410*GLRLM_LRHGLE. All three features are from contrast-enhanced T1-weighted sequence with the formulae used in their calculation shown in Additional file 4.

In the matched training cohort, 3-year and 5-year FFS were $68.2 \%(95 \% \mathrm{CI}=0.60-0.77)$ vs. $63.7 \%(95 \% \mathrm{CI}=$ $0.56-0.73)$ and $59.4 \%(95 \% \mathrm{CI}=0.51-0.70)$ vs. $59.0 \%$ (95\% CI $=0.51-0.69)$ for ICT+CCRT and CCRT alone patients, respectively $(\mathrm{HR}=0.85,95 \% \mathrm{CI}=0.57-1.27$, $p=0.44$; Fig. 3a). Among patients, 116.2 (46.9\%) had high ICTOS while 131.6 (53.1\%) had low ICTOS. Note that the patient numbers here are not integers because they are weighted numbers of the matched training cohort after IPTW. ICTOS could predict treatment outcome for the additional ICT $\left(p_{\text {interaction }}<0.001\right)$. In the high ICTOS group, 3-year and 5-year FFS were 69.3\% $(95 \% \mathrm{CI}=0.59-0.82)$ and $64.0 \%(95 \% \mathrm{CI}=0.53-0.78)$ in $\mathrm{ICT}+\mathrm{CCRT}$ subgroup and $45.6 \%(95 \% \mathrm{CI}=0.34-0.61)$
Original training cohort

263 retrospectively included

(with events or more than 3 years of follow-up) 126 received ICT + CCRT 137 received CCRT

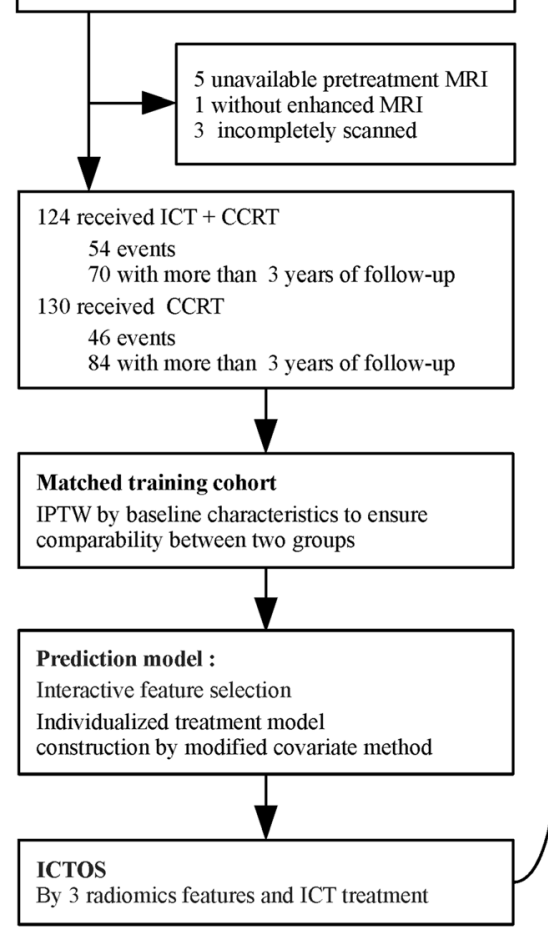

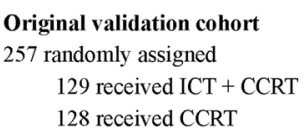

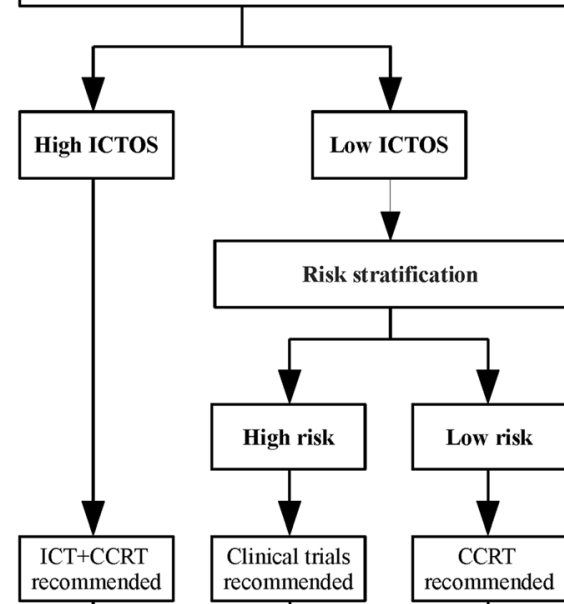

Fig. 2 Study design. The belief design of patient recruitment, matching procedures, model construction and validation, and further stratification analysis. ICT, induction chemotherapy; CCRT, concurrent chemoradiotherapy; MRI, magnetic resonance imaging; IPTW, inverse probability of treatment weighting; ICTOS, Induction Chemotherapy Outcomes Score 
and $36.5 \%(95 \% \mathrm{CI}=0.25-0.53)$ in CCRT alone subgroup $(\mathrm{HR}=0.43,95 \% \mathrm{CI}=0.25-0.74, p=0.002$, Fig. $3 \mathrm{~b})$. Therefore, in the high ICTOS group, there were $23.7 \%$ absolute benefit at 3-year in favor of ICT+CCRT vs. CCRT alone, and $27.5 \%$ absolute benefit at 5-year. While in the low ICTOS group, 3-year and 5-year FFS were $67.1 \%(95 \% \mathrm{CI}=0.57-0.80)$ and $55.8 \%(95 \% \mathrm{CI}=$ 0.44-0.71) in the ICT+CCRT subgroup and $78.7 \%$ (95\% $\mathrm{CI}=0.70-0.89)$ and $76.5 \%(95 \% \mathrm{CI}=0.67-0.87)$ in the CCRT alone subgroup $(\mathrm{HR}=1.78,95 \% \mathrm{CI}=0.96-3.31$, $p=0.063$; Fig. 3c).

In the validation cohort, the last follow-up was on $\mathrm{Au}$ gust 31, 2018 [6]. The median follow-up was 69.5 months (IQR 61.9-76.9). The 3-year and 5-year FFS were $82.4 \%$ $(95 \% \mathrm{CI}=0.76-0.89)$ vs. $69.9(95 \% \mathrm{CI}=0.62-0.79)$, and $80.0 \%(95 \% \mathrm{CI}=0.73-0.87)$ vs. $63.4 \%(95 \% \mathrm{CI}=0.55-0.73)$ for ICT+CCRT and CCRT alone patients, respectively $(\mathrm{HR}=0.51,95 \% \mathrm{CI}=0.32-0.83, p=0.006,12.5 \%$ and $16.6 \%$ absolute benefit at 3 and 5 years in favor of ICT+CCRT vs. CCRT alone; Fig. 3d). ICTOS identification ability was validated in our validation cohort $\left(p_{\text {interaction }}=0.019\right)$. In the high ICTOS group ( $n=73 / 248), 3$-year and 5-year FFS were $89.7 \%(95 \% \mathrm{CI}=0.81-1.00)$ and $89.7 \%(95 \% \mathrm{CI}=$ $0.81-1.00)$ in ICT+CCRT; and $61.8 \%(95 \% \mathrm{CI}=0.47-0.81)$ and $52.8 \%(95 \% \mathrm{CI}=0.38-0.73)$ in CCRT alone subgroups $(\mathrm{HR}=0.17,95 \% \mathrm{CI}=0.06-0.51, p<0.001$, Fig. 3e). Therefore, in the high ICTOS group, there were $27.9 \%$ absolute benefit at 3-year in favor of ICT+CCRT vs. CCRT alone, and $36.9 \%$ absolute benefit at 5-year. While in the low ICTOS group $(n=175 / 248)$, 3-year and 5-year FFS were $79.1 \%(95 \% \mathrm{CI}=0.71-0.88)$ and $75.5 \%(95 \% \mathrm{CI}=0.67-$ $0.85)$ in the ICT+CCRT, and $73.0 \%(95 \% \mathrm{CI}=0.64-0.83)$ and $67.4 \%(95 \% \mathrm{CI}=0.58-0.78)$ in the CCRT alone subgroups $(\mathrm{HR}=0.75,95 \% \mathrm{CI}=0.43-1.31, p=0.31$; Fig. 3f).

Clinical risk factors did not show consistently significant associations with ICTOS across training and validation

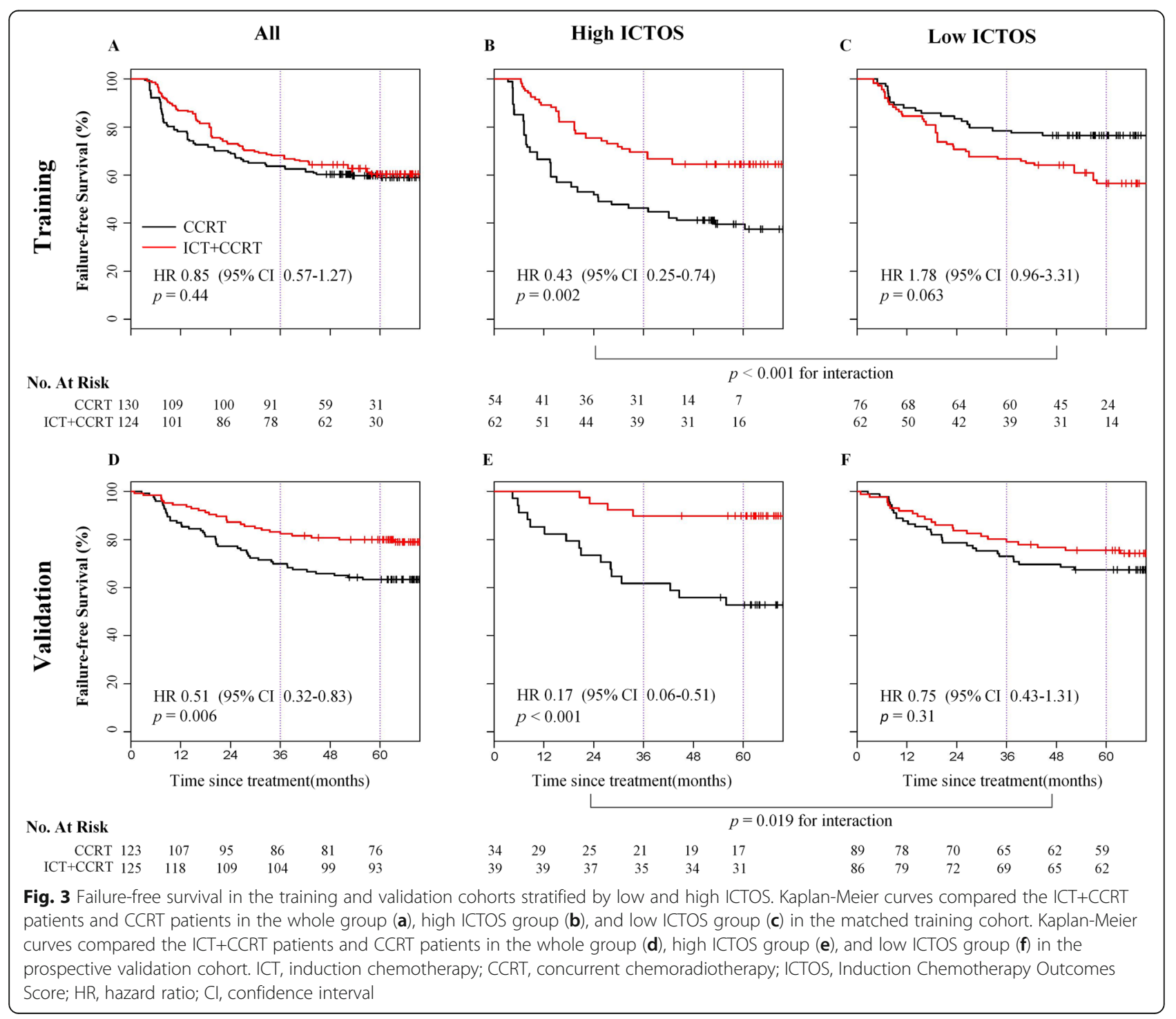


cohorts (Table 1). Furthermore, with secondary endpoints (OS, D-FFS, and LR-FFS), ICTOS also successfully selected patients who could benefit most from additional ICT (Additional file 7: Figure S3), demonstrating the good generalizability of our model. The interaction between additional ICT and prognostic factors including pEBV DNA ( $<2000$ vs. $\geq 2000$ copy/ml), N stage (N1 vs. N2-3), and $\mathrm{T}$ stage (T1-3 vs. T4) was not significant in the matched training $\left(p_{\text {interaction }}=0.73\right.$ for $\mathrm{pEBV}$ DNA, $p_{\text {interac- }}$ tion $=0.21$ for $\mathrm{N}$ stage, $p_{\text {interaction }}=0.071$ for $\mathrm{T}$ stage) and validation $\left(p_{\text {interaction }}=0.74\right.$ for $\mathrm{pEBV}$ DNA, $p_{\text {interaction }}=$ 0.47 for $\mathrm{N}$ stage, $p_{\text {interaction }}=0.77$ for $\mathrm{T}$ stage) cohorts
(Additional file 8: Figure S4). Moreover, in an interaction analysis for pEBV DNA with 1500, 4000, and $6000 \mathrm{copy} / \mathrm{ml}$ as cutoff points, all $p_{\text {interaction values remained non- }}$ significant. These results illustrate that these factors were not predictive of treatment outcome for the additional ICT despite some being prognostic factors [22].

Since low ICTOS patients in the validation cohort could not gain significant FFS improvement from the additional ICT, it was not possible to conclude that CCRT alone was sufficient in patients with unsatisfactory 5-year FFS $(71.4 \%, 95 \% \mathrm{CI}=0.65-0.78)$. Therefore, we further explored the low ICTOS group by conducting

Table 1 Association of baseline characteristics with ICTOS in both training and validation sets

\begin{tabular}{|c|c|c|c|c|c|c|}
\hline & Training set & & & & & \\
\hline & Low ICTOS $(n=138)$ & High ICTOS $(n=116)$ & $P$ value & Low ICTOS $(n=175)$ & High ICTOS $(n=73)$ & $P$ value \\
\hline Age & & & 0.06 & 0.49 & & 0.49 \\
\hline$<42$ years & 45 (32.6) & $52(44.8)$ & & $81(46.3)$ & $38(52.1)$ & \\
\hline$\geq 42$ years & $93(67.4)$ & $64(55.2)$ & & $94(53.7)$ & $35(47.9)$ & \\
\hline Sex & & & 0.02 & & & 0.40 \\
\hline Male & $109(79.0)$ & $75(64.7)$ & & $144(82.3)$ & $56(76.7)$ & \\
\hline Female & $29(21.0)$ & $41(35.3)$ & & $31(17.7)$ & $17(23.3)$ & \\
\hline Staging ${ }^{a}$ & & & 0.04 & & & 0.80 \\
\hline III & $84(70.9)$ & $55(47.4)$ & & $95(65.7)$ & $46(73.0)$ & \\
\hline IV & $54(39.1)$ & $61(52.6)$ & & $60(34.3)$ & $27(37.0)$ & \\
\hline T stage $^{a}$ & & & 0.21 & & & 0.33 \\
\hline $\mathrm{T} 1$ & $4(2.9)$ & $2(1.7)$ & & $5(2.9)$ & $1(1.4)$ & \\
\hline $\mathrm{T} 2$ & $14(10.1)$ & $7(6.0)$ & & $8(4.6)$ & $5(6.8)$ & \\
\hline T3 & $82(59.4)$ & $62(53.4)$ & & $114(65.1)$ & $40(54.8)$ & \\
\hline T4 & $38(27.5)$ & $45(38.8)$ & & $48(27.4)$ & $27(37.0)$ & \\
\hline N stage ${ }^{a}$ & & & 0.48 & 0.08 & & 0.08 \\
\hline N1 & $66(47.8)$ & $47(40.5)$ & & $98(56.0)$ & $36(49.3)$ & \\
\hline N2 & $50(36.2)$ & $46(39.7)$ & & $62(35.4)$ & $35(47.9)$ & \\
\hline N3 & $22(15.9)$ & $23(19.8)$ & & $15(8.6)$ & $2(2.7)$ & \\
\hline Cervical nodal necrosis & & & 0.90 & & & 0.32 \\
\hline No & 99 (71.7) & 85 (73.3) & & $114(65.1)$ & $53(72.6)$ & \\
\hline Yes & $39(28.3)$ & $31(26.7)$ & & $61(34.9)$ & $20(27.4)$ & \\
\hline Primary tumor volume & $<0.001$ & & $<0.001$ & 0.56 & & 0.56 \\
\hline$<34 \mathrm{ml}$ & $97(70.3)$ & $53(45.7)$ & & $88(50.3)$ & $33(45.2)$ & \\
\hline$\geq 34 \mathrm{ml}$ & $41(29.7)$ & $63(54.3)$ & & $87(49.7)$ & $40(54.8)$ & \\
\hline Pretreatment pEBV DNA level & & & 0.17 & & & 0.08 \\
\hline$<2000 \mathrm{copy} / \mathrm{ml}$ & $75(54.3)$ & $52(44.8)$ & & $60(45.7)$ & $24(32.9)$ & \\
\hline$\geq 2000 \mathrm{copy} / \mathrm{ml}$ & $63(45.7)$ & $64(55.2)$ & & $95(54.3)$ & $49(67.1)$ & \\
\hline Chemoradiotherapy regimen & & & 0.22 & & & 0.64 \\
\hline $\mathrm{ICT}+\mathrm{CCRT}$ & $62(44.9)$ & $62(53.4)$ & & $86(49.1)$ & $39(53.4)$ & \\
\hline CCRT & $76(55.1)$ & $54(46.6)$ & & $89(50.9)$ & $34(46.6)$ & \\
\hline
\end{tabular}

ICTOS Induction Chemotherapy Outcomes Score, pEBV DNA plasma Epstein-Barr Virus DNA, CCRT concurrent chemoradiotherapy, ICT, induction chemotherapy aStaging, T classification, and $\mathrm{N}$ classification were based on the 7th edition of the American Joint Committee on Cancer/International Union Against Cancer staging systems 
risk stratification. $\mathrm{N}$ stage, $\mathrm{T}$ stage, primary tumor volume, and pEBV DNA were confirmed as stratification factors (see detailed results in Additional file 13: Table S4). To facilitate clinical application, patients with more than two high-risk factors were stratified into the highrisk group, and others into the low-risk group. Low-risk group patients had significantly higher 3-year and 5-year FFS than high-risk group $(90.4 \%$ [ $95 \% \mathrm{CI}=0.84-0.97]$ vs. 65.7\% [95\% CI $=0.57-0.76], 86.3 \%[95 \% \mathrm{CI}=0.79-0.95]$ vs. $60.8 \%$ [95\% CI $=0.52-0.71]$; $\mathrm{HR}=1.77,95 \% \mathrm{CI}=1.26-$ $2.47, p<0.001$, Fig. 4a). In both groups, there was no significant difference in survival curves between ICT+CCRT and CCRT alone patients (low-risk group, $\mathrm{HR}=1.08,95 \%$ $\mathrm{CI}=0.33-3.54, p=0.90$; high-risk group, $\mathrm{HR}=0.72,95 \%$ $\mathrm{CI}=0.38-1.35, p=0.30$, Fig. $4 \mathrm{~b})$, which confirmed the robustness of our model in different subgroups.

\section{Discussion}

In this relatively large-cohort study, we developed an individualized MRI biomarker, ICTOS, which could predict treatment outcome for additional ICT. The constructed biomarker showed impressive performance in the validation cohort from a prospective randomized controlled trial (NCT01245959). Moreover, in comparison to the widely used clinical risk factors, our proposed biomarker was more effective. Furthermore, combining the clinical prognostic factors with ICTOS, we built CDSS, which might predict the subgroups that would benefit most from ICT+CCRT or CCRT alone.

Interestingly, our model successfully identified patients who would benefit most from ICT+CCRT in the prospective validation cohort; high ICTOS patients from
ICT+CCRT subgroup had nearly 90\% 5-year FFS with $36.9 \%$ absolute benefit, whereas, without ICTOS identification, ICT+CCRT group had $80.0 \%$ 5-year FFS with a $16.6 \%$ absolute benefit.

According to NCCN guideline, ICT+CCRT is recommended for NPC patients with stages II-IVa (accounting for $>90 \%$ of all non-metastasis NPC) [23]. However, there was no biomarker discovered for selecting patients who could benefit most from ICT+CCRT based on individual tumor characteristics. Indeed, the development of truly predictive (as opposed to prognostic) treatment response biomarker is a difficult proposition, which ideally requires balanced datasets from treated versus untreated patients. Our previous randomized clinical trials were perfectly suited for this purpose. Our results showed that additional ICT could improve FFS in high, but not in low, ICTOS patients. The result indicated that high ICTOS patients benefited the most from additional ICT; thus, ICT+CCRT should be recommended for them. Our MRI radiomic-based ICTOS, the first validated imaging biomarker developed to predict treatment outcome for therapy in NPC, is potentially a useful tool clinically, for specifically selecting patients for ICT.

Three 2D radiomic features (skewness, GLCM_variance, and GLRLM_LRHGLE) were finally selected to construct ICTOS to predict benefits from additional ICT. Interestingly, all three features were extracted from contrastenhanced T1 MR images profiling the perfusion and permeability of tumor vasculature. This finding suggests that tumor angiogenesis might be the predominant factor for the true therapeutic gain, which is in accordance with preliminary studies on mechanism of tumor resistance to

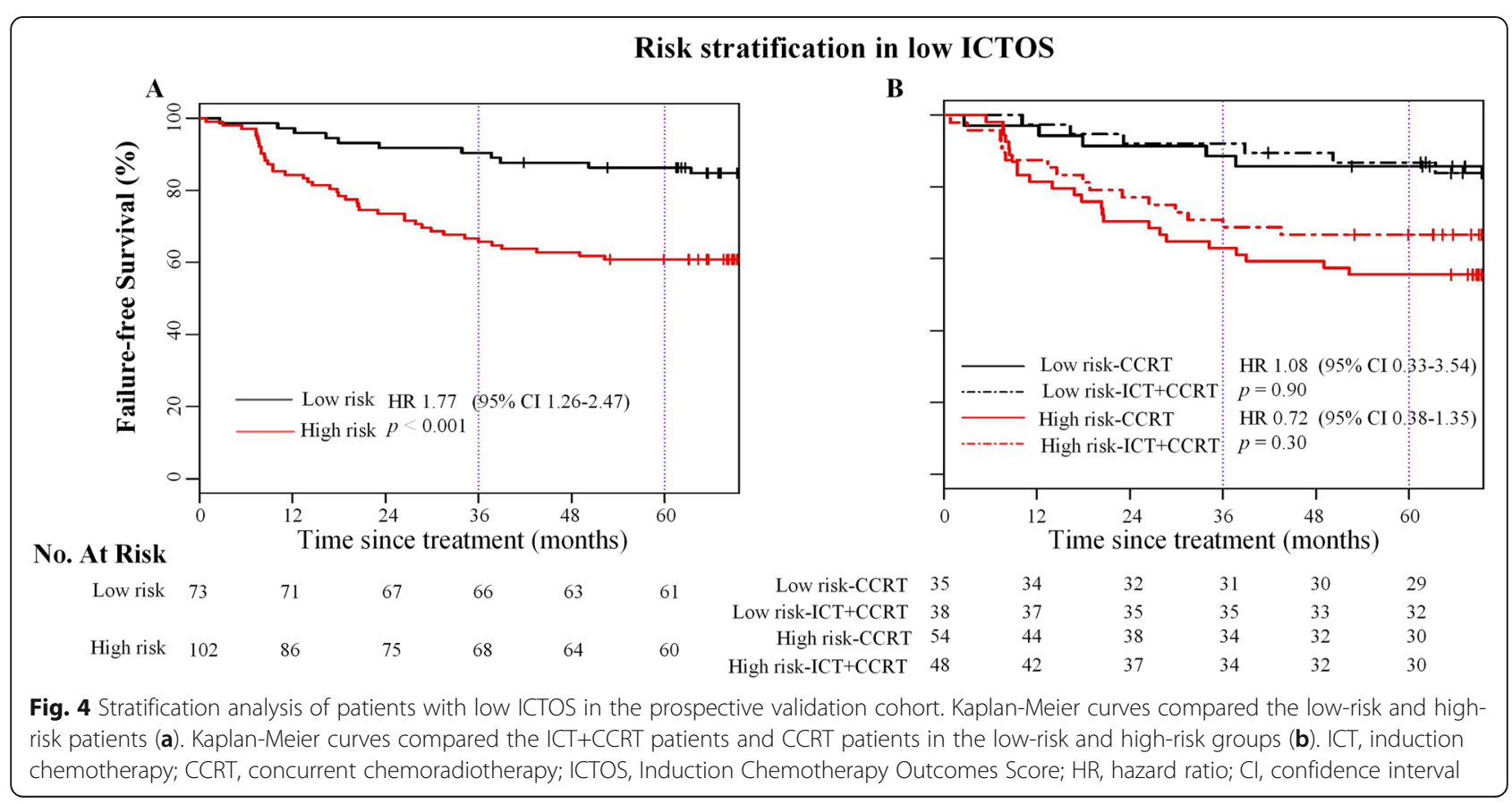


chemotherapy [24]. We selected several typical images, with different levels of the three radiomic features and attempted surmising the biologic mechanism of these image features (Additional file 9: Figure S5). Skewness indicates the bias in contrast enhancement correlating with vasculature density, and thus blood supply, suggesting that patients with low ICTOS might have impaired drug delivery, resulting in ineffective ICT [24]. Conversely, the remaining two features represent tumor vasculature heterogeneity. Low ICTOS suggested a high heterogeneity in the structure and function of tumor vasculature. This might lead to a more hostile and complex tumor microenvironment with hypoxia, acidosis, and promotion of the tumor population towards more chemo-resistant one, therefore compromising ICT efficacy [24]. Our findings are also consistent with features found in a previous study predicting the short-term response to ICT in NPC [25]. A preliminary trial (RTOG 0615) [26] showed a promising response by adding bevacizumab to standard chemoradiation in LANPC. The spatially explicit analysis of radiomic feature to tumor vasculature, the mechanism of tumor angiogenesis to chemo-resistance, and the confirmation of the chemotherapeutic gain from induction of vessel normalization in NPC still warrants further investigation.

As a key classification of the anatomical extent of tumor, the current TNM staging system reflecting tumor size ( $\mathrm{T}$ stage), lymph node status ( $\mathrm{N}$ stage), and metastasis status ( $\mathrm{M}$ stage) is the only tool to guide patient selection for ICT in NPC [27]. In our study, prognostic factors including pEBV DNA, $\mathrm{N}$ stage, and $\mathrm{T}$ stage were not predictive of treatment outcome for the additional ICT. The above prognostic factors were mainly related to tumor burden, but not intrinsic chemosensitivity. In this study, only LANPC patients were included; obviously, the difference in tumor load among patients, being relatively slight, might be concealed by more powerful treatment response difference from our imaging biomarker; hence, these prognostic factors in the LANPC could not distinguish who benefited most from ICT.

Our subgroup analysis showed that patients with low ICTOS and high risk had the worst prognosis (5year FFS rate in these patients was only $60.8 \%$ ). Possible reasons included advanced $\mathrm{N}$ and $\mathrm{T}$ stages, as well as large tumor volume, which were all proved to be negative prognostic factors. In this subgroup, the introduction of ICT was ineffective and the treatment outcome with CCRT alone was poor. Thus, the patients in this subgroup might need intensification of therapy or new therapies, such as anti-angiogenesis [26], metronomic adjuvant chemotherapy [28], and immunotherapy [29]. While for the patients with low ICTOS and low risk, CCRT alone already had a good 5 -year FFS of $86.3 \%$ and the introduction of ICT generated no significant benefit. Therefore, CCRT alone might be preferred for patients in this subgroup to avoid unnecessary ICT-related expense and toxicity, as well as the prolonged wait before definitive radiotherapy. In conclusion, our CDSS, which combined radiomic ICTOS with clinical prognostic factors, could classify advanced locoregional NPC into three groups with appropriate recommendations for individual therapies as follows: (1) high ICTOS: ICT+CCRT, (2) low ICTOS and low risk: CCRT alone, and (3) low ICTOS and high risk: clinical trials. This is the first time that clinical prognostic and predictive factors have been integrated to guide the individualized treatment of NPC. However, further validation in larger population is still required for the latter two recommendations.

The generalizability of this imaging biomarker is a major concern in clinical practice. For NPC patients, MRI is a routine image method for staging, because of its superiority in soft-tissue contrast. Via target delineation in radiotherapy, segmentation of tumor is unlikely to bring extra burden to clinical practice. Moreover, the imaging biomarker can be automatically generated simply by importing ICTOS-based module into treatment planning system. Besides, through the stratification analysis, our imaging biomarker was not affected by clinical factors like stage and pEBV DNA, showing the potential for generalization. We have deposited our biomarker and CDSS into open access (www.radiomics.net.cn/platform.html) to facilitate the validation and application.

Our study had some limitations. First, due to the retrospective nature of the training set, inevitably, there was possible selection bias between the two treatment groups in the training cohort. This could also be found when comparing the survival results between the training and validation cohorts (Fig. 3a, d), the higher event rate of ICT group in training cohort than the validation cohort. However, this high event rate in the training cohort allowed us to train the ICTOS with a small number of patients. Moreover, validation in the subset of a randomized controlled dataset indicated the robustness of ICTOS. Second, due to the limitation of the data size, further investigations in additional independent cohorts are necessary. For example, larger and multi-center cohorts, as well as our ongoing trials with another regimen of ICT (NCT01872962, gemcitabine and cisplatin), could provide potential validation cohorts to test the reproducibility and universality of ICTOS. Third, our previous work found that the expression of 13 genes [30] predicted the prognosis and efficacy of CCRT in LANPC. Other studies proposed proteins, such as SQSTM1 [21] and genes, such as ERCC1 [31] to predict chemotherapy response in NPC. In the following study, we plan to incorporate the molecular profile based on the same 
settings (LANPC patients) to confirm the current findings. In our study, many different MR scanners and MR acquisition protocols were used; how this influenced the radiomic features and ICTOS model should be studied further in the future.

In conclusion, the proposed imaging biomarker (ICTOS) provided a robust, feasible, and economical solution to identify patients who were more likely to gain a survival benefit from ICT+CCRT, which could influence clinical decisions such as the indication for ICT treatment.

\section{Supplementary information}

Supplementary information accompanies this paper at https://doi.org/10. 1186/s12916-019-1422-6.

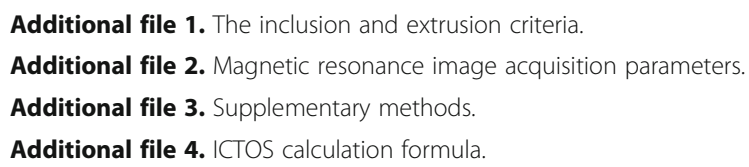
treatment weighting between ICT+CCRT and CCRT patient groups. Abbreviations: CCRT, concurrent chemoradiotherapy; ICT, induction chemotherapy; IPTW, Inverse Probability of Treatment Weighting.

Additional file 6: Figure S2. Analysis of radiomic features' robustness. Abbreviations: T1, T1-weighted images; T2, T2-weighted images; T1C, contrast enhanced T1-weighted images; ICC, inter-class correlation coefficient. Note: An ICC greater than 0.8 indicates good consistency. As the figure shown, a large majority of features achieve this standard, which affirm the reproducibility of imaging features.

Additional file 7: Figure S3. Kaplan-Meier survival curves with secondary endpoints for the two treatment groups in the validation cohort. Abbreviations: ICT, induction chemotherapy; CCRT, concurrent chemoradiotherapy; ICTOS, Induction Chemotherapy Outcomes Score; $\mathrm{HR}$, hazard ratio; $\mathrm{Cl}$, confidence interval. Note: Because there was no patient with distance failure in the high ICTOS group, relative measurements could not be calculated (E).

Additional file 8: Figure S4. Failure-free survival in the validation cohort stratified by the usual clinical risk factors. Abbreviations: $I C$, induction chemotherapy; CCRT, concurrent chemoradiotherapy; pEBV DNA, plasma Epstein-Barr Virus DNA. Note: $P_{\text {interaction }}=0.74$ for $p E B V$ DNA, $P_{\text {interaction }}$

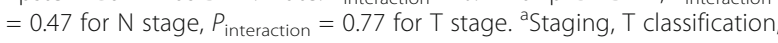
$\mathrm{N}$ classification were determined based on the 7th edition of the American Joint Commission on Cancer staging system.

Additional file 9: Figure S5. MR images of patients with high ICTOS and low ICTOS. Abbreviations: MR, Magnetic Resonance; ICTOS, Induction Chemotherapy Outcomes Score. Note: Contrast enhanced T1 MRI images in a patient with high ICTOS (skewness = -2.02; GLRLM_LRHGLE = 4.58; GLCM_variance $=-1.26$, ICTOS $=3.80$, Figure A); and a patient with low ICTOS (skewness $=1.82 ;$ GLRLM_LRHGLE $=-1.46 ;$ GLCM_variance $=1.37$, ICTOS $=-2.49$, Fig. B).

Additional file 10: Table S1. MR imaging sequences.

Additional file 11: Table S2. Acquisitions parameters of axial CE-T1W FSE in the training and validation cohorts.

Additional file 12: Table S3. Standardized differences in the training sets after weighting by propensity score.

Additional file 13: Table S4. Univariate analyses of risk factors in patients with low ICTOS $(n=175)$.

\section{Abbreviations}

CCRT: Concurrent chemoradiotherapy; CDSSs: Clinical decision support systems; Cl: Confidence interval; CR: Complete response; D-FFS: Distant failure-free survival; FFS: Failure-free survival; GLCM_variance: Variance from Gray-Level Co-occurrence Matrix; GLRLM_LRHGLE: Long Run High Gray Level Emphasis from Gray-Level Run-Length matrix; HR: Hazard ratio; ICT: Induction chemotherapy; ICTOS: Induction Chemotherapy Outcome Score;

IPTW: Inverse probability of treatment weighting; IQR: Interquartile range; LANPC: Locoregionally advanced nasopharyngeal carcinoma; LRFFS: Locoregional failure-free survival; MR: Magnetic resonance imaging; NCCN: National Comprehensive Cancer Network; NPC: Nasopharyngeal carcinoma; OS: Overall survival; PACS: Picture archiving and communication system; pEBV: Plasmas Epstein-Barr virus; ROI: Region of interest; SD: Stable disease

\section{Acknowledgements}

We thanked Professor Ai-Hua Lin from the Department of Medical Statistics and Epidemiology, School of Public Health, Sun Yat-sen University, Guangzhou, China, for her review of the statistical methodology of this study.

\section{Authors' contributions}

LLT, DD, FZ, and LZZ conceived, designed, and supervised the project. DD, $F Z$, LZZ, MJF, CLH, and JJY contributed to the design of the study, writing the protocol, and data preparation, analysis, and interpretation. DD, FZ, and LZZ drafted the manuscript. LLT, JM, JT, and YS performed the quality assessment and revised the manuscript. All authors have read and approved the submitted version.

\section{Funding}

This work was supported by grants from the Natural Science Foundation of Guangdong Province (2017A030312003), National Key R\&D Program of China (2017YFA0205200, 2017YFC1308700, 2017YFC1309100), National Natural Science Foundation of China $(81572962,81971776,81771924,81501616$, $81227901,81671851,81527805$, and 61671449), Health \& Medical Collaborative Innovation Project of Guangzhou City, China (201803040003), Innovation Team Development Plan of the Ministry of Education (IR_17R110), the Beijing Natural Science Foundation (L182061), and the Youth Innovation Promotion Association CAS (2017175).

The funding organizations had no role in the design and conduct of the study; collection, management, analysis, and interpretation of the data; preparation, review, or approval of the manuscript; and decision to submit the manuscript for publication.

\section{Availability of data and materials}

The datasets used and/or analyzed during the current study are available from the corresponding author on reasonable request.

\section{Ethics approval and consent to participate}

This study was approved by the Institutional Ethical Review Boards of the Sun Yat-sen University Cancer Center, and the requirement for informed consent was waived.

\section{Consent for publication}

Not applicable.

\section{Competing interests}

The authors declare that they have no competing interests.

\section{Author details}

${ }^{1}$ CAS Key Laboratory of Molecular Imaging, Institute of Automation, Chinese Academy of Sciences, No. 95 Zhongguancun East Road, Hai Dian District, Beijing 100190, People's Republic of China. ${ }^{2}$ Department of Radiation oncology, State Key Laboratory of Oncology in South China; Collaborative Innovation Center for Cancer Medicine, Sun Yat-sen University Cancer Center, 651 Dongfeng Road East, Guangzhou 510060, People's Republic of China. ${ }^{3}$ School of Artificial Intelligence, University of Chinese Academy of Sciences, Beijing 100049, People's Republic of China. ${ }^{4}$ Department of Radiotherapy, The Fifth Affiliated Hospital, Sun Yat-sen University, Zhuhai 519000, People's Republic of China. ${ }^{5}$ Beijing Advanced Innovation Center for Big Data-Based Precision Medicine, School of Medicine, Beihang University, Beijing 100191, People's Republic of China. 
Received: 10 March 2019 Accepted: 9 September 2019

Published online: 23 October 2019

\section{References}

1. Torre LA, Bray F, Siegel RL, Ferlay J, Lortet-Tieulent J, Jemal A. Global cancer statistics, 2012. CA Cancer J Clin. 2015;65(2):87-108.

2. Pan JJ, Ng WT, Zong JF, Chan LL, O'Sullivan B, Lin SJ, Sze HC, Chen YB, Choi HC, Guo QJ. Proposal for the 8th edition of the AJCC/UICC staging system for nasopharyngeal cancer in the era of intensity-modulated radiotherapy. Cancer. 2016;122(4):546-58.

3. National Comprehensive Cancer Network guidelines. 2018. https:/www.nccn.org/ professionals/physician_gls/pdf/head-and-neck.pdf Accessed 15 Feb 2018. 2018.

4. Chen YP, Tang LL, Yang Q, Poh SS, Hui EP, Chan ATC, Ong WS, Tan T, Wee J, Li WF, et al. Induction chemotherapy plus concurrent chemoradiotherapy in endemic nasopharyngeal carcinoma: individual patient data pooled analysis of four randomized trials. Clin Cancer Res. 2018;24(8):1824-33.

5. Sun Y, Li WF, Chen NY, Zhang N, Hu GQ, Xie FY, Sun Y, Chen XZ, Li JG, Zhu $X D$. Induction chemotherapy plus concurrent chemoradiotherapy versus concurrent chemoradiotherapy alone in locoregionally advanced nasopharyngeal carcinoma: a phase 3, multicentre, randomised controlled trial. Lancet Oncol. 2016;17(11):1509-20.

6. Li WF, Chen NY, Zhang N, Hu GQ, Xie FY, Sun Y, Chen XZ, Li JG, Zhu XD, Hu CS, et al. Concurrent chemoradiotherapy with/without induction chemotherapy in locoregionally advanced nasopharyngeal carcinoma: longterm results of phase 3 randomized controlled trial. Int J Cancer. 2019; 145(1):295-305.

7. Peng H, Chen L, Li WF, Guo R, Mao YP, Zhang Y, Guo Y, Sun Y, Ma J. Tumor response to neoadjuvant chemotherapy predicts long-term survival outcomes in patients with locoregionally advanced nasopharyngeal carcinoma: a secondary analysis of a randomized phase 3 clinical trial. Cancer. 2017;123(9):1643-52.

8. De Sousa EMF, Vermeulen L, Fessler E, Medema JP. Cancer heterogeneity--a multifaceted view. EMBO Rep. 2013;14(8):686-95.

9. Lin JC, Wang WY, Chen KY, Wei YH, Liang WM, Jan JS, Jiang RS. Quantification of plasma Epstein-Barr virus DNA in patients with advanced nasopharyngeal carcinoma. N Engl J Med. 2004;350(24):2461-70.

10. Liu N, Chen NY, Cui RX, Li WF, Li Y, Wei RR, Zhang MY, Sun Y, Huang BJ, Chen $\mathrm{M}$, et al. Prognostic value of a microRNA signature in nasopharyngeal carcinoma: a microRNA expression analysis. Lancet Oncol. 2012;13(6):633-41.

11. Lambin P, Leijenaar RTH, Deist TM, Peerlings J, de Jong EEC, van Timmeren J, Sanduleanu S, Larue R, Even AJG, Jochems A, et al. Radiomics: the bridge between medical imaging and personalized medicine. Nat Rev Clin Oncol. 2017;14(12):749-62.

12. Dong D, Tang L, Li ZY, Fang MJ, Gao JB, Shan XH, Ying XJ, Sun YS, Fu J, Wang $X X$, et al. Development and validation of an individualized nomogram to identify occult peritoneal metastasis in patients with advanced gastric cancer. Ann Oncol. 2019;30(3):431-8.

13. Peng $H$, Dong D, Fang MJ, Li L, Tang LL, Chen L, Li WF, Mao YP, Fan W, Liu $L Z$, et al. Prognostic value of deep learning PET/CT-based radiomics: potential role for future individual induction chemotherapy in advanced nasopharyngeal carcinoma. Clin Cancer Res. 2019;25(14):4271-9.

14. Song J, Shi J, Dong D, Fang M, Zhong W, Wang K, Wu N, Huang Y, Liu Z, Cheng $Y$, et al. A new approach to predict progression-free survival in stage IV EGFR-mutant NSCLC patients with EGFR-TKI therapy. Clin Cancer Res. 2018;24(15):3583-92

15. Austin PC, Stuart EA. Moving towards best practice when using inverse probability of treatment weighting (IPTW) using the propensity score to estimate causal treatment effects in observational studies. Stat Med. 2015; 34(28):3661-79.

16. Zwanenburg ALS, Vallières M. Image biomarker standardisation initiative. arXiv preprint arXiv. 2016:1612.07003.

17. Aerts HJ, Velazquez ER, Leijenaar RT, Parmar C, Grossmann P, Carvalho S, Bussink J, Monshouwer R, Haibe-Kains B, Rietveld D, et al. Decoding tumour phenotype by noninvasive imaging using a quantitative radiomics approach. Nat Commun. 2014;5:4006

18. Yang L, Dong D, Fang M, Zhu Y, Zang Y, Liu Z, Zhang H, Ying J, Zhao X, Tian J. Can CT-based radiomics signature predict KRAS/NRAS/BRAF mutations in colorectal cancer? Eur Radiol. 2018;28(5):2058-67.

19. Tian L, Alizadeh AA, Gentles AJ, Tibshirani R. A simple method for estimating interactions between a treatment and a large number of covariates. J Am Stat Assoc. 2014;109(508):1517-32.
20. Zhao SG, Chang SL, Spratt DE, Erho N, Yu M, Ashab HA-D, Alshalalfa M, Speers C, Tomlins SA, Davicioni E, et al. Development and validation of a 24-gene predictor of response to postoperative radiotherapy in prostate cancer: a matched, retrospective analysis. Lancet Oncol. 2016;17(11):1612-20.

21. Yang Q, Zhang MX, Zou X, Liu YP, You R, Yu T, Jiang R, Zhang YN, Cao JY, Hong $\mathrm{MH}$, et al. A prognostic bio-model based on SQSTM1 and N-stage identifies nasopharyngeal carcinoma patients at high risk of metastasis for additional induction chemotherapy. Clin Cancer Res. 2018;24(3):648-58.

22. Paik S, Tang G, Shak S, Kim C, Baker J, Kim W, Cronin M, Baehner FL, Watson $D$, Bryant J, et al. Gene expression and benefit of chemotherapy in women with node-negative, estrogen receptor-positive breast cancer. J Clin Oncol. 2006;24(23):3726-34

23. Tang LL, Chen YP, Mao YP, Wang ZX, Guo R, Chen L, Tian L, Lin AH, Li L, Sun $Y$, et al. Validation of the 8th edition of the UICC/AJCC staging system for nasopharyngeal carcinoma from endemic areas in the intensitymodulated radiotherapy era. J Natl Compr Cancer Netw. 2017;15(7):913-9.

24. Carmeliet $P$, Jain RK. Principles and mechanisms of vessel normalization for cancer and other angiogenic diseases. Nature Reviews Dug Discovery. 2011; 10(6):417-27.

25. Wang G, He L, Yuan C, Huang Y, Liu Z, Liang C. Pretreatment MR imaging radiomics signatures for response prediction to induction chemotherapy in patients with nasopharyngeal carcinoma. Eur Radiol. 2018;98:100-6.

26. Lee NY, Zhang Q, Pfister DG, Kim J, Garden AS, Mechalakos J, Hu K, Le QT, Colevas AD, Glisson BS, et al. Addition of bevacizumab to standard chemoradiation for locoregionally advanced nasopharyngeal carcinoma (RTOG 0615): a phase 2 multi-institutional trial. Lancet Oncol. 2012;13(2):172-80.

27. Peng $H$, Chen L, Zhang J, Li WF, Mao YP, Zhang Y, Liu LZ, Tian L, Lin AH, Sun $Y$, et al. Induction chemotherapy improved long-term outcomes of patients with locoregionally advanced nasopharyngeal carcinoma: a propensity matched analysis of 5-year survival outcomes in the era of intensity-modulated radiotherapy. J Cancer. 2017;8(3):371-7.

28. Twu CW, Wang WY, Chen CC, Liang KL, Jiang RS, Wu CT, Shih YT, Lin PJ, Liu YC, Lin JC. Metronomic adjuvant chemotherapy improves treatment outcome in nasopharyngeal carcinoma patients with postradiation persistently detectable plasma Epstein-Barr virus deoxyribonucleic acid. Int J Radiat Oncol Biol Phys. 2014;89(1):21-9.

29. Li J, Chen QY, He J, Li ZL, Tang XF, Chen SP, Xie CM, Li YQ, Huang LX, Ye $\mathrm{SB}$, et al. Phase I trial of adoptively transferred tumor-infiltrating lymphocyte immunotherapy following concurrent chemoradiotherapy in patients with locoregionally advanced nasopharyngeal carcinoma. Oncoimmunology. 2015;4(2):e976507.

30. Tang XR, Li YQ, Liang SB, Jiang W, Liu F, Ge WX, Tang LL, Mao YP, He QM, Yang $X J$, et al. Development and validation of a gene expression-based signature to predict distant metastasis in locoregionally advanced nasopharyngeal carcinoma: a retrospective, multicentre, cohort study. Lancet Oncol. 2018;19(3):382-93.

31. Huang PY, Li Y, Mai HQ, Luo RZ, Cai YC, Zhang L. Expression of ERCC1 predicts clinical outcome in locoregionally advanced nasopharyngeal carcinoma treated with cisplatin-based induction chemotherapy. Oral Oncol. 2012;48(10):964-8

\section{Publisher's Note}

Springer Nature remains neutral with regard to jurisdictional claims in published maps and institutional affiliations.
Ready to submit your research? Choose BMC and benefit from:
- fast, convenient online submission
- thorough peer review by experienced researchers in your field
- rapid publication on acceptance
- support for research data, including large and complex data types
- gold Open Access which fosters wider collaboration and increased citations
- maximum visibility for your research: over $100 \mathrm{M}$ website views per year
At BMC, research is always in progress.
Learn more biomedcentral.com/submissions 